PROCEEDINGS OF THE

AMERICAN MATHEMATICAL SOCIETY

Volume 126, Number 10, October 1998, Pages 3125-3130

S 0002-9939(98)04418-9

\title{
A RESULT ON THE GELFAND-KIRILLOV DIMENSION OF REPRESENTATIONS OF CLASSICAL GROUPS
}

\author{
CHEN-BO ZHU
}

(Communicated by Roe Goodman)

\begin{abstract}
Let $\left(G, G^{\prime}\right)$ be the reductive dual pair $(O(p, q), S p(2 n, \mathbb{R}))$. We show that if $\pi$ is a representation of $S p(2 n, \mathbb{R})$ (respectively $O(p, q)$ ) obtained from duality correspondence with some representation of $O(p, q)$ (respectively $S p(2 n, \mathbb{R}))$, then its Gelfand-Kirillov dimension is less than or equal to $(p+q)\left(2 n-\frac{p+q-1}{2}\right)\left(\right.$ respectively $\left.2 n\left(p+q-\frac{2 n+1}{2}\right)\right)$.
\end{abstract}

\section{Gelfand-Kirillov dimension of $\mathcal{U}(\mathfrak{g})$ Modules}

We shall recall some notions in this section ([V], [KL]).

Let $k$ be a field. A $k$-algebra is called almost commutative if there exists a filtration $A_{0} \subseteq A_{1} \subseteq \cdots$ such that

(i) $A_{0}=k$.

(ii) $A_{1}$ is finite dimensional, and $A$ is generated as an algebra by $A_{1}$.

(iii) The associated graded algebra $\operatorname{gr}(A)=\sum_{i=0}^{\infty} A_{i} / A_{i-1}$ is commutative.

Let $A$ be a $k$-algebra which is almost commutative with respect to the filtration $\mathcal{A}=\left(A_{i}\right)_{i=0}^{\infty}$, and let $V$ be a graded $A$ module with a finite filtration $\mathcal{V}=\left(V_{i}\right)_{i=0}^{\infty}$ such that the associated graded module $\operatorname{gr}(V)$ of $\operatorname{gr}(A)$ is finitely generated. Then the function $\operatorname{dim}_{k} V_{i}$ is a polynomial in $i$ for sufficiently large $i$, called the HilbertSamuel polynomial. The degree of this polynomial is independent of the choice of filtrations of $A$ and $V$, and is called the Gelfand-Kirillov dimension of $V$, or GKdim of $V$ for short.

Now let $\mathfrak{g}$ be a Lie algebra over $\mathbb{C}$. Let $A=\mathcal{U}(\mathfrak{g})$, the universal enveloping algebra of $\mathfrak{g}$, with the standard filtration given by $A_{i}=\mathcal{U}(\mathfrak{g})_{i}$, the subspace of $\mathcal{U}(\mathfrak{g})$ spanned by monomials of the form $X_{1} \cdots X_{m}$, with $m \leq i$ and $X_{j} \in \mathfrak{g}$, for each $j$. By the Poincare-Birkhoff-Witt theorem, the associated graded algebra $\operatorname{gr}(\mathcal{U}(\mathfrak{g}))$ is canonically isomorphic to $S(\mathfrak{g})$, the symmetric algebra of $\mathfrak{g}$ - a polynomial algebra in $\operatorname{dim} \mathfrak{g}$ variables. Suppose $V$ is a finitely generated $\mathcal{U}(\mathfrak{g})$ module. Choose a finite dimensional generating subspace $V_{0}$, and set $V_{i}=\mathcal{U}(\mathfrak{g})_{i} V_{0}$. Then $V$ becomes a graded $\mathcal{U}(\mathfrak{g})$ module, and so the degree of the polynomial $\operatorname{dim}_{\mathbb{C}} V_{i}$ (for $i$ sufficiently large) gives the Gelfand-Kirillov dimension of $V$. It is a non-negative integer less than or equal to $\operatorname{dim} \mathfrak{g}$.

Received by the editors November 4, 1996 and, in revised form, February 27, 1997.

1991 Mathematics Subject Classification. Primary 22E46, 22E47.

Key words and phrases. Classical groups, duality correspondence, Gelfand-Kirillov dimension.

(C)1998 American Mathematical Society 


\section{REPRESENTATIONS FROM DUAL PAIR CORRESPONDENCE}

Let $V=\mathbb{R}^{p+q}$ be equipped with the following non-degenerate symmetric form $($,$) of signature (p, q)$ :

$$
(x, y)=x^{t} I_{p, q} y, \quad x, y \in \mathbb{R}^{p+q},
$$

where $I_{p, q}=\left(\begin{array}{cc}I_{p} & 0 \\ 0 & -I_{q}\end{array}\right)$. Let $G=O(p, q)$ be its isometry group. Denote $W=$ $V^{2 n}$, the direct sum of $2 n$ copies of $V$. We may identify $W$ with the vector space $M_{p+q, 2 n}(\mathbb{R})$ of $(p+q) \times 2 n$ real matrices. It is easy to see that $W$ admits a symplectic structure given by

$$
\left\langle W_{1}, W_{2}\right\rangle=\operatorname{tr}\left(W_{1}^{t} I_{p, q} W_{2} J_{n}\right), \quad W_{1}, W_{2} \in M_{p+q, 2 n}(\mathbb{R}),
$$

where $J_{n}=\left(\begin{array}{cc}0 & I_{n} \\ -I_{n} & 0\end{array}\right), t r$ denotes the trace function on the space of $2 n \times 2 n$ real matrices. Let $S p$ be the group of symplectic transformations of $W$.

Let $G^{\prime}=S p(2 n, \mathbb{R})=\left\{g \in G L(2 n, \mathbb{R}) \mid g J_{n} g^{t}=J_{n}\right\}$. Then $G$ and $G^{\prime}$ will act on $M_{p+q, 2 n}(\mathbb{R})$ via matrix multiplication on the left and on the right. Moreover we have a reductive dual pair $[\mathrm{H} 1]$

$$
\left(G, G^{\prime}\right)=(O(p, q), S p(2 n, \mathbb{R})) \subset S p .
$$

Let $\tilde{S} p$ be the metaplectic cover of $S p$, and for any subgroup $E$ of $S p$, let $\tilde{E}$ be the inverse image of $E$ in $\tilde{S} p$.

Let $H$ be either $G$ or $G^{\prime}$, and $H^{\prime}$ the other member.

Theorem 1. Let $\pi$ be a representation of $\tilde{H}$ obtained from duality correspondence with $\tilde{H}^{\prime}([\mathrm{H} 3])$.

a) Let $H=S p(2 n, \mathbb{R})$; then

Gelfand-Kirillov dimension of $\pi \leq \begin{cases}(p+q)\left(2 n-\frac{p+q-1}{2}\right), & \text { if } p+q \leq 2 n, \\ n(2 n+1), & \text { if } p+q>2 n .\end{cases}$

b) Let $H=O(p, q)$; then

$$
\text { Gelfand-Kirilliov dimension of } \pi \leq \begin{cases}2 n\left(p+q-\frac{2 n+1}{2}\right), & \text { if } 2 n \leq p+q, \\ \frac{(p+q)(p+q-1)}{2}, & \text { if } 2 n>p+q .\end{cases}
$$

Remarks. 1) By a result of Vogan (Proposition 5.7, [V]), the Gelfand-Kirillov dimension of any irreducible admissible $\tilde{H}$ module $\pi$ is less than or equal to the dimension of a maximal unipotent subgroup. Thus in case a) where $H=S p(2 n, \mathbb{R})$, it implies that the Gelfand-Kirillov dimension of $\pi$ is at most $n^{2}$. So the second part and part of the first part of our inequality are weaker than this general result of Vogan. We thank the referee for pointing out this. However our proof is very elementary, and the main interest here is in the cases where $p+q$ is small relative to $n$. Similar remarks apply to case b).

2) Analogous results hold for other reductive dual pairs $\left(G, G^{\prime}\right) \subset S p$. The needed adjustment in the argument is very minor, and that is to apply appropriate result from classical invariant theory as in [W]. We shall leave this to the interested reader. 


\section{Proof of Theorem 1}

Recall that $H$ is either $G=O(p, q)$ or $G^{\prime}=S p(2 n, \mathbb{R})$. Let $\mathfrak{h}$ be the Lie algebra of $H$, and $\mathfrak{h}_{\mathbb{C}}$ be its complexification. Here and after we use the convention of indicating the appropriate complexification by a subscript $\mathbb{C}$.

Let $\omega$ be the oscillator representation of $\tilde{S} p([\mathrm{H} 2])$. Recall that $\mathcal{U}\left(\mathfrak{h}_{\mathbb{C}}\right)$ is the universal enveloping algebra of $\mathfrak{h}_{\mathbb{C}}$ endowed with the standard filtration. Then $\omega\left(\mathcal{U}\left(\mathfrak{h}_{\mathbb{C}}\right)\right)$ inherits a corresponding filtration. Let $\operatorname{gr}\left(\omega\left(\mathcal{U}\left(\mathfrak{h}_{\mathbb{C}}\right)\right)\right)$ be the graded algebra associated with this filtration. One version of the First Main Theorem of Classical Invariant Theory [H1] asserts the isomorphism

$$
\operatorname{gr}\left(\omega\left(\mathcal{U}\left(\mathfrak{h}_{\mathbb{C}}\right)\right)\right) \cong P\left(V_{\mathbb{C}}^{2 n}\right)^{H_{\mathbb{C}}^{\prime}}
$$

where $P\left(V_{\mathbb{C}}^{2 n}\right)$ denotes the polynomial algebra on $V_{\mathbb{C}}^{2 n}=W_{\mathbb{C}}$, and $P\left(V_{\mathbb{C}}^{2 n}\right)^{H_{\mathbb{C}}^{\prime}}$ is the algebra of $H_{\mathbb{C}}^{\prime}$ invariants in $P\left(V_{\mathbb{C}}^{2 n}\right)$.

Proposition 1. Let $\pi$ be a representation of $\tilde{H}$ obtained from duality correspondence with $\tilde{H}^{\prime}$. Then the Gelfand-Kirillov dimension of $\pi$ is less than or equal to the Gelfand-Kirillov dimension of $P\left(V_{\mathbb{C}}^{2 n}\right)^{H_{\mathbb{C}}^{\prime}}$.

Proof. From [H3], we know that $\pi$ is a finitely generated, admissible quotient of $\left.\omega\right|_{\tilde{H}^{\prime}}$. Let $\mathcal{H}_{\pi}$ be the space of $\tilde{K}$-finite vectors of $\pi$, where $K$ is a maximal compact subgroup of $H$. Then $\mathcal{H}_{\pi}$ is a module for $\mathcal{U}\left(\mathfrak{h}_{\mathbb{C}}\right)$, and we have a surjective homomorphism

$$
\omega\left(\mathcal{U}\left(\mathfrak{h}_{\mathbb{C}}\right)\right) \rightarrow \pi\left(\mathcal{U}\left(\mathfrak{h}_{\mathbb{C}}\right)\right)
$$

Define a filtration on $\pi\left(\mathcal{U}\left(\mathfrak{h}_{\mathbb{C}}\right)\right)$ by

$$
\pi\left(\mathcal{U}\left(\mathfrak{h}_{\mathbb{C}}\right)\right)_{i}=\pi\left(\mathcal{U}\left(\mathfrak{h}_{\mathbb{C}}\right)_{i}\right)
$$

Clearly we have a commutative diagram

$$
\begin{aligned}
\omega\left(\mathcal{U}\left(\mathfrak{h}_{\mathbb{C}}\right)\right) & \rightarrow \pi\left(\mathcal{U}\left(\mathfrak{h}_{\mathbb{C}}\right)\right) \\
\downarrow & \downarrow \\
\operatorname{gr} \omega\left(\mathcal{U}\left(\mathfrak{h}_{\mathbb{C}}\right)\right) & \longrightarrow \operatorname{gr} \pi\left(\mathcal{U}\left(\mathfrak{h}_{\mathbb{C}}\right)\right) .
\end{aligned}
$$

We therefore have a surjective homomorphism

$$
P\left(V_{\mathbb{C}}^{2 n}\right)^{H_{\mathbb{C}}^{\prime}} \cong \operatorname{gr} \omega\left(\mathcal{U}\left(\mathfrak{h}_{\mathbb{C}}\right)\right) \rightarrow \operatorname{gr} \pi\left(\mathcal{U}\left(\mathfrak{h}_{\mathbb{C}}\right)\right) .
$$

This clearly implies that the Gelfand-Kirillov dimension of $\pi$ is less than or equal to the Gelfand-Kirillov dimension of $P\left(V_{\mathbb{C}}^{2 n}\right)^{H_{\mathbb{C}}^{\prime}}$.

Proposition 2. a) Let $H=S p(2 n, \mathbb{R})$; then the Gelfand-Kirillov dimension of $P\left(V_{\mathbb{C}}^{2 n}\right)^{H_{\mathbb{C}}^{\prime}}$ is

$$
\begin{cases}(p+q)\left(2 n-\frac{p+q-1}{2}\right), & \text { if } p+q \leq 2 n \\ n(2 n+1), & \text { if } p+q>2 n\end{cases}
$$

b) Let $H=O(p, q)$; then the Gelfand-Kirillov dimension of $P\left(V_{\mathbb{C}}^{2 n}\right)^{H_{\mathbb{C}}^{\prime}}$ is

$$
\begin{cases}2 n\left(p+q-\frac{2 n+1}{2}\right), & \text { if } 2 n \leq p+q \\ \frac{(p+q)(p+q-1)}{2}, & \text { if } 2 n>p+q\end{cases}
$$


Proof. Since $P\left(V_{\mathbb{C}}^{2 n}\right)^{H_{\mathbb{C}}^{\prime}}$ is a commutative algebra, its Gelfand-Kirillov dimension is the same as the Krull dimension $([\mathrm{KL}])$. In turn it is equal to the transcendence degree of $P\left(V_{\mathbb{C}}^{2 n}\right)^{H_{\mathbb{C}}^{\prime}}$, since it is an integral domain and finitely generated.

First let $H=S p(2 n, \mathbb{R})$ so that $H^{\prime}=O(p, q)$. We complexify the symmetric bilinear form $($,$) on V$ to a symmetric linear form on $V_{\mathbb{C}}$, which we still denote by $($,$) . Let m=p+q$.

Let $Z=\left(z_{1}, \ldots, z_{2 n}\right) \in V_{\mathbb{C}}^{2 n}$ be a typical element. For $1 \leq i, j \leq 2 n$, let $r_{i, j}(Z)=$ $\left(z_{i}, z_{j}\right)=z_{i}^{t} I_{p, q} z_{j}$. Then $r_{i, j} \in P\left(V_{\mathbb{C}}^{2 n}\right)^{H_{\mathbb{C}}^{\prime}}$. The First Main Theorem of Classical Invariant Theory for orthogonal groups $[\mathrm{W}]$ states that $P\left(V_{\mathbb{C}}^{2 n}\right)^{H_{\mathbb{C}}^{\prime}}$ is generated by $r_{i, j}$, where $1 \leq i \leq j \leq 2 n$.

Consider the $2 n \times 2 n$ symmetric matrix

$$
R=Z^{t} I_{p, q} Z=\left(\begin{array}{ccc}
r_{1,1}(Z) & \cdots & r_{1,2 n}(Z) \\
\cdots & \cdots & \cdots \\
r_{2 n, 1}(Z) & \cdots & r_{2 n, 2 n}(Z)
\end{array}\right)
$$

Observe that for each specialization of $Z$, the rank of $R$ is less than or equal to $m$. Thus the determinant of any minor of size $(m+1) \times(m+1)$ of $R$ is equal to zero, namely we have

$$
\operatorname{det}\left(\begin{array}{ccc}
r_{i_{1}, j_{1}} & \cdots & r_{i_{1}, j_{m+1}} \\
\cdots & \cdots & \cdots \\
r_{i_{m+1}, j_{1}} & \cdots & r_{i_{m+1}, j_{m+1}}
\end{array}\right)=0
$$

where $1 \leq i_{1}<\cdots<i_{m+1} \leq 2 n$, and $1 \leq j_{1}<\cdots<j_{m+1} \leq 2 n$. In fact the Second Main Theorem of Classical Invariant Theory asserts that these generate all the relations among the generators $r_{i, j} \in P\left(V_{\mathbb{C}}^{2 n}\right)^{H_{\complement}^{\prime}}$, but we shall not use the full force of this result.

Suppose $m \leq 2 n$. Let

$$
B=\left\{r_{i, j} \mid 1 \leq i \leq j \leq 2 n, i \leq m\right\} .
$$

In view of the Second Main Theorem of Classical Invariant Theory, we see that $B$ is an algebraically independent set. Moreover by taking $\left(i_{1}, \ldots, i_{m}, i_{m+1}\right)=$ $(1, \ldots, m, m+1),\left(j_{1}, \ldots, j_{m}, j_{m+1}\right)=(1, \ldots, m, m+k)$, where $1 \leq k \leq n-2 m$, the determinant relations in Equation (1) imply that $r_{m+1, m+k}$ is in the field of fractions generated by $B$. Thus $r_{m+1, j}$ is in this field for any $j$. Continuing this way, we see that any $r_{i, j} \notin B$ is in the field of fractions of $B$. Thus the transcendence degree of $P\left(V_{\mathbb{C}}^{2 n}\right)^{H_{\mathbb{C}}^{\prime}}$ is the cardinality of $B$, which is $m\left(2 n-\frac{m-1}{2}\right)$.

Suppose $m>2 n$. Then the $n(2 n+1)$ generators of $P\left(V_{\mathbb{C}}^{2 n}\right)^{H_{\mathbb{C}}^{\prime}}$, namely $\left\{r_{i, j} \mid 1 \leq\right.$ $i \leq j \leq 2 n\}$, are algebraically independent, and so the transcendence degree of $P\left(V_{\mathbb{C}}^{2 n}\right)^{H_{\mathbb{C}}^{\prime}}$ is $n(2 n+1)$.

Now let $H=O(p, q)$ so that $H^{\prime}=S p(2 n, \mathbb{R})$. We shall argue similarly as in the above case. Again we identify $V_{\mathbb{C}}^{2 n}$ with $M_{m, 2 n}(\mathbb{C})$. Let $U=\left(\begin{array}{c}u_{1} \\ \vdots \\ u_{m}\end{array}\right) \in$ $M_{m, 2 n}(\mathbb{C})$ be a typical element. Thus $u_{1}, \ldots, u_{m}$ are the row vectors of $U$. For $1 \leq i, j \leq m$, let $s_{i, j}(U)=u_{i} J_{n} u_{j}^{t}$. Then $s_{i, j} \in P\left(M_{m, 2 n}(\mathbb{C})\right)^{S p(2 n, \mathbb{C})}$. The First Main Theorem of Classical Invariant Theory for symplectic groups [W] states that $P\left(M_{m, 2 n}(\mathbb{C})\right)^{S p(2 n, \mathbb{C})}$ is generated by $s_{i, j}$, where $1 \leq i<j \leq m$. 
Consider the $m \times m$ skewsymmetric matrix

$$
S=U J_{n} U^{t}=\left(\begin{array}{ccc}
s_{1,1}(U) & \cdots & s_{1, m}(U) \\
\cdots & \cdots & \cdots \\
s_{m, 1}(U) & \cdots & s_{m, m}(U)
\end{array}\right) .
$$

Observe that for each specialization of $U$, the rank of $S$ is less than or equal to $2 n$. Thus the Pfaffian of any principal minor of size $(2 n+2) \times(2 n+2)$ of $S$ is equal to zero, namely we have

$$
\operatorname{Pf}\left(\begin{array}{ccc}
s_{i_{1}, i_{1}} & \cdots & s_{i_{1}, i_{2 n+2}} \\
\cdots & \cdots & \cdots \\
s_{i_{2 n+2}, i_{1}} & \cdots & s_{i_{2 n+2}, i_{2 n+2}}
\end{array}\right)=0,
$$

where $1 \leq i_{1}<\cdots<i_{2 n+2} \leq m$.

Suppose $2 n \leq m$. Let

$$
C=\left\{s_{i, j} \mid 1 \leq i<j \leq m, i \leq 2 n\right\} .
$$

In view of relations of $s_{i, j}$ 's given in the Second Main Theorem of Classical Invariant Theory [W], we see that $C$ is an algebraically independent set. Moreover by taking $\left(i_{1}, i_{2}, \ldots, i_{2 n+1}, i_{2 n+2}\right)=(1,2, \ldots, 2 n+1,2 n+k)$, where $2 \leq k \leq m-2 n$, the Pfaffian relations in Equation (2) imply that $s_{2 n+1,2 n+k}$ is in the field of fractions generated by $C$. Thus $s_{2 n+1, j}$ is in this field for any $j$. Continuing this way, we see that any $s_{i, j} \notin C$ is in the field of fractions of $C$. Thus the transcendence degree of $P\left(M_{m, 2 n}(\mathbb{C})\right)^{S p(2 n, \mathbb{C})}$ is the cardinality of $C$, which is $2 n\left(m-\frac{2 n+1}{2}\right)$.

Suppose $2 n>m$. Then the $\frac{m(m-1)}{2}$ generators of $P\left(M_{m, 2 n}(\mathbb{C})\right)^{S p(2 n, \mathbb{C})}$, namely $\left\{s_{i, j} \mid 1 \leq i<j \leq m\right\}$, are algebraically independent, and so the transcendence degree of $P\left(M_{m, 2 n}(\mathbb{C})\right)^{S p(2 n, \mathbb{C})}$ is $\frac{m(m-1)}{2}$.

\section{Concluding REMARKS}

In the course of works on some representations obtained from duality correspondence, the author and his collaborators have computed the Gelfand-Kirillov dimension of these representations. We list a few results here.

a) Let $q=0$, and let $\pi$ be a representation of $\tilde{S} p(2 n, \mathbb{R})$ obtained from the duality correspondence of the pair $(O(p), S p(2 n, \mathbb{R}))$. Thus $\pi$ is a holomorphic representation of $\tilde{S} p(2 n, \mathbb{R})$. Then GKdim of $\pi=p\left(n-\frac{p-1}{2}\right)$ if $p \leq n$, and GKdim of $\pi=\frac{n(n+1)}{2}$ if $p>n$. See [TZ].

b) Let $p+q \leq n$, namely $(O(p, q), S p(2 n, \mathbb{R}))$ is a dual pair in the stable range with $O(p, q)$ the small member $([\mathrm{H} 5])$, and let $\pi$ be the representation of $\tilde{S} p(2 n, \mathbb{R})$ corresponding to the trivial representation of $O(p, q)$. Then GKdim of $\pi=(p+q)\left(n-\frac{p+q-1}{2}\right)$. See [LZ2].

c) Let $p+q$ is even and $2 n \leq \min (p, q)$, namely $(O(p, q), S p(2 n, \mathbb{R}))$ is a dual pair in the stable range with $S p(2 n, \mathbb{R})$ the small member, and let $\pi$ be the representation of $O(p, q)$ corresponding to the trivial representation of $S p(2 n, \mathbb{R})$. Then GKdim of $\pi=n(p+q-2 n-1)$. See $[\mathrm{ZH}]$.

The above examples suggest that when the size of $H^{\prime}$ is very small relative to that of $H$, the Gelfand-Kirillov dimension of a representation $\pi$ of $\tilde{H}$ obtained from duality correspondence with a representation $\sigma$ of $\tilde{H}^{\prime}$ may be independent of $\sigma$. They also indicate that our estimate of the Gelfand-Kirillov dimension is not sharp. This is due to the fact that given a finite dimensional generating subspace 
$V_{0}(\S 0)$, the map $\pi\left(\mathcal{U}\left(\mathfrak{h}_{\mathbb{C}}\right)_{i}\right) \otimes V_{0} \mapsto \pi\left(\mathcal{U}\left(\mathfrak{h}_{\mathbb{C}}\right)_{i}\right) V_{0}$ often has a kernel. For example in case a), if we take $V_{0}$ to be the lowest $\tilde{U}(n)$-type of the holomorphic representation $\pi$ of $\tilde{S} p(2 n, \mathbb{R})$, then

$$
\pi\left(\mathcal{U}\left(\mathfrak{h}_{\mathbb{C}}\right)_{i}\right) V_{0}=\pi\left(S\left(\mathfrak{p}^{+}\right)_{i}\right) V_{0},
$$

where $S\left(\mathfrak{p}^{+}\right)$is the symmetric algebra of $\mathfrak{p}^{+}$, and $\mathfrak{h}_{\mathbb{C}}=\mathfrak{k} \oplus \mathfrak{p}^{+} \oplus \mathfrak{p}^{-}$is the HarishChandra decomposition of $\mathfrak{h}_{\mathbb{C}}=\mathfrak{s p}(2 n, \mathbb{C})$.

Finally we remark on the methods the Gelfand-Kirillov dimension is computed in these examples. In case a), we use equation (3) and then apply invariant theoretic considerations, and so the argument is quite similar to the one given in this article. In cases b) and c), our computation of the Gelfand-Kirillov dimension relies on an explicit description of $\tilde{K}$-types of the representations concerned, where $K$ is a maximal compact subgroup. It is worthwhile to mention that they are all $\tilde{K}$ multiplicity-free in cases b) and c).

\section{BiBLIOGRAPHIES}

[A] J. Adams, The theta correspondence over $\mathbb{R}$, Preprint, Workshop at the University of Maryland (1994).

[BK] W. Borho and H. Kraft, Uber die Gelfand-Kirillov-dimension, Math. Ann. 220 (1976), 1-24. MR 54:367

[H1] R. Howe, Remarks on classical invariant theory, Trans. Amer. Math. Soc. 313 (1989), 539-570. MR 90h:22015a

[H2] $\quad \theta$-series and invariant theory, Proceedings of Symposia in Pure Mathematics 33 (1979), 275-285. MR 81f:22034

[H3] , Transcending classical invariant theory, J. Amer. Math. Soc. 2 (1989), 535-552. MR 90k:22016

[H4] - Dual pairs in physics: Harmonic oscillators, photons, electrons, and singletons, Lectures in Appl. Math., Vol. 21, Amer. Math. Soc., Providence, R.I. (1985), 179- 206. MR 86:22036

[H5] $-L^{2}$ duality for stable reductive dual pairs, preprint.

[KL] G.R. Krause and T.H. Lenegan, Growth of algebras and Gelfand-Kirillov dimension, Research Notes in Mathematics, V 116, Pitman Publishing Inc., 1985. MR 86g:16001

[L1] J.S. Li, On the singular rank of a representation, Proc. Amer. Math. Soc. 106 (2) (1989), 567-571. MR 89k:22029

[L2] Singular unitary representations of classical groups, Invent. Math. 97 (1989), 237255. MR 90h:22021

[LZ1] S.T. Lee and C.B. Zhu, Degenerate principal series and local theta correspondence, to appear in the Trans. Amer. Math. Soc. CMP 97:11

[LZ2] _ Degenerate principal series and local theta correspondence II, Israel J. Math. 100 (1997), 29-59. CMP 97:11

[TZ] E.C. Tan and C.B. Zhu, Poincare series of holomorphic representations, Indag. Mathem. N.S. 7 (1) (1996), 111-126.

[V] D. Vogan, Gelfand-Kirillov dimension for Harish-Chandra modules, Invent. Math. 48 (1978), 75-98. MR 58:22205

[W] H. Weyl, The classical groups, Princeton University Press, Princeton, New Jersey, 1946.

$[\mathrm{ZH}]$ C.B. Zhu and J.S. Huang, On certain small representations of indefinite orthogonal groups, Representation Theory 1 (1997), 190-206. CMP 97:14

Department of Mathematics, National University of Singapore, Kent Ridge, SingaPORE 119260

E-mail address: matzhucb@leonis.nus.sg.edu 